\title{
Interactive comment on "Rupture-dependent breakdown energy in fault models with thermo-hydro-mechanical processes" by Valère Lambert and Nadia Lapusta
}

Valère Lambert and Nadia Lapusta

vlambert@caltech.edu

Received and published: 5 October 2020

Thank you for your assessment of our work and helpful comments. Please find responses to each comment/question in the attached supplemental pdf. For clarity, we have reiterated each comment/question, colored in blue, with our responses in black.

Please also note the supplement to this comment:

https://se.copernicus.org/preprints/se-2020-115/se-2020-115-AC3-supplement.pdf 